\title{
Adverse pregnancy outcomes in extremely maternal age*
}

\author{
Saifon Chawanpaiboon ${ }^{\#}$, Pattaya Hengrasmee
}

Department of Obstetrics \& Gynaecology, Faculty of Medicine Siriraj Hospital, Mahidol University, Bangkok, Thailand

Email: ${ }^{\text {siscw@mahidol.ac.th }}$

Received 19 February 2013; revised 21 March 2013; accepted 30 March 2013

Copyright (C) 2013 Saifon Chawanpaiboon, Pattaya Hengrasmee. This is an open access article distributed under the Creative Commons Attribution License, which permits unrestricted use, distribution, and reproduction in any medium, provided the original work is properly cited.

\begin{abstract}
Objective: To study the adverse outcome in pregnant women $\leq 16$ and $\geq 40$ years. Study Design: $A$ total of 1100 cases, 1061 cases of pregnant women $\leq 16$ years, 20 - 29 and $\geq 40$ years with completed charts between 1st January, 2006 to 31st December, 2010, were enrolled in this study. The patient data includeing demographic data, hospital course, maternal laboratory investigations, maternal complications, placental complications and neonatal outcomes were recorded. SPSS version 14, one-way Anowa, Chi-square and analysis of varience were used to compare the data among two and three groups. Results: Anemia, underlying medical diseases including heart, thyroid and pulmonary diseases, obstetric complications including severe pre-eclampsia and gestational diabetes mellitus, placenta previa and preterm labour; medical administrations including magnesium sulfate, dexamethasone and bricanyl, outcome of deliveries and neonatal outcomes, were different with statistical significance between the 3 groups of pregnant women. Conclusion: Adverse maternal and neonatal outcomes were mostly found in the pregnant women $\leq 16$ and $\geq$ 40 years. Education of contraception, safe sex and effective care during pregnancy of both extremely maternal age groups should be implemented to reduce those poor outcomes.
\end{abstract}

Keywords: Adverse Pregnancy Outcome; Extremely Maternal Age; Young Maternal Age; Advanced Maternal Age

\section{INTRODUCTION}

The advanced revolution of social, culture and technology in many developing countries around the world results in different education and economic status among

*Conflict of interest: There was no conflict of interest.

${ }^{\#}$ Corresponding author. people. Poor education is usually found in teenage pregnancy and high education is not surprised to relate with advanced maternal age. Teenage pregnancy continues to be a major health problem in Thailand and other developing countries around the world [1]. Around $85 \%$ of teenage pregnancies are in developing world [1] and $25 \%$ of mothers are in this age group [2]. Thailand has the second highest rate of teenage pregnancy in the world which may result from the environmental degradation, poor family income, less educated population and lack of warmth and care from family. Therefore the importance of the issue of teenage pregnancy is increased. Most of Thai teenagers have unintended pregnancies because of the lack of knowledge about sex and pregnancy prevention.

There was a study about teenage pregnancy and adverse neonatal outcomes in Canada which studied pregnant women at age 20 years or less $(2.6 \%$ of all first pregnancy). The study found that those with first pregnancy at age 20 years or less had an increased risk of 4.5 times of preterm delivery than those at age $20-39$ years. Moreover, the risk of fetal birth defects and infant mortality in teenage pregnancy were as high as 1.8 and 3.8 times, respectively [3].

On the contrary, high education results in delayed pregnancy in women. Recent advances in assisted reproductive technology make it possible for advanced age women to conceive. Therefore the life birth rate for advanced age women has increased. The impact of advanced maternal age and delayed childbearing on pregnancy outcomes is controversial.

However, there was no study about adverse pregnancy outcome of extremely maternal age in Siriraj Hospital therefore this research was performed. The authors hope that the results of this research would be beneficial for further improvement of the care in extremely maternal age.

\section{METHODS}

This study was approved by Siriraj Ethics Committee of 
the Faculty of Medicine Siriraj Hospital, COA no. Si 431/2011. The study design was a retrospective cohort study. The sample size, using a power and precision analysis formula was calculated by the incidence of preterm labour, gestational hypertension, gestational diabetes mellitus, cesarean section, anemia, low birth weight infant fetal anomalies and admission of infants to the neonatal intensive care unit (NICU) at Siriraj Hospital. The highest sample size which was calculated from NICU admission of infants about 1100 cases was used.

The completed data of patients between 1st January, 2006 and 31st December, 2010, were enrolled in this study. During this 7-year period, all completed charts of maternal age $\leq 16$ years included 1061 cases while those of maternal age of $20-29$ and $\geq 40$ years were 20,776 and 1319 cases, respectively. Systematic sampling of the charts in maternal age of $20-29$ and $\geq 40$ years was performed. Running checks from hospital numbers, one of every 18 medical records $(1,19,37,56, \cdots, 20,681)$ was collected and one of every 6 medical records $(7,13$, $19, \cdots, 1314)$ was deleted, respectively. Therefore the total numbers of the charts in maternal age of $20-29$ and $\geq 40$ years, 1100 cases, were recruited.

The patient data including demographic data, hospital course, maternal investigation and complications, placental complications and neonatal outcomes were recorded in the data record form.

\subsection{Definition of Adverse Outcomes in Mother}

- Anemia means pregnant women with hemoglobin concentration below 11.0 grams/dL or hematocrit below $33 \%$ [4].

- High blood pressure during pregnancy means that people with systolic blood pressure $\geq 140 \mathrm{mmHg}$ or diastolic blood pressure $\geq 90 \mathrm{mmHg}$ for 2 more times when measured at least 6 hours apart [5].

- Diabetes mellitus is diagnosed by the criteria of abnormal glucose tolerance test. The pregnant women must have a screening test with 50 gram 1 hour challenge test. The positive screening test will be followed by a diagnostic 100 grams oral glucose tolerance test (OGTT). The normal blood glucose level at $0,1,2,3$ hours are not greater than 105, 190, 165, $145 \mathrm{mg} / \mathrm{dL}$, respectively, If they are abnormal more than two values, so it would have been diagnosed with gestational diabetes mellitus (GDM) in pregnant women. The pregnant women who can control blood glucose level to normal by dietary means alone are diagnosed as GDMA1. If insulin therapy is considered, the pregnant women are diagnosed with GDMA2 [6].

- Placental abruption means the separation of placenta from attachment before delivery of the baby [7].
- Placenta previa means the placental attachment cover or near the internal cervical os [7].

- Preterm birth means the birth between gestational age between $24+0$ to $36+6$ weeks [8].

\subsection{Definition of Adverse Outcomes in Neonate}

- Neonatal deaths [9]

- Stillbirth means no vital signs of the baby during or after birth.

- Early neonatal death means death of a baby during the first 7 days after birth.

- Late neonatal death means the death of infants from 8 - 28 days after birth.

- Low birth weight means neonatal birth weight less than 2500 grams [9].

- Congenital abnormalities are defined as neonates with structural congenital anomalies.

- NICU admission means newborns treated in the neonatal intensive care unit.

SPSS version 14 was used to analyze data. One-way Anowa and Chi-square were used to compare the categorical data and analysis of variance was used to compare the continuous variable data among two groups. Results were reported as means, standard deviations (SD) or percentages. Subgroup analysis was also performed. The level of statistical significance (p-value) was $<0.05$.

\section{RESULTS}

During the period of 1st January to 31st December, 2010, all completed charts of 1061 cases of maternal age $\leq 16$ years and randomized completed charts of 1100 cases of maternal age $20-29$ and $\geq 40$ years were collected. Demographic data including body mass index, gravida, parity and abortion among the 3 groups were different with statistical significance (Table 1). Only 946 from 1061 cases; and 1010 and 1028 from 1100 cases of maternal age $\leq 16$ years; and $20-29$ and $\geq 40$ years were collected, respectively. The missing cases were those of birth before admission and those who had no antenatal visit (Figures 1-3). The number of patients who were anti-HIV positive, had anemia and alpha-thalassemia among the 3 groups were different with statistical significance (Table 1). Only 1007 (29.2\%) from 1061; and $1082(24.3 \%)$ and $1093(21.0 \%)$ cases from 1100 cases of the patients with thalassemia were collected. The missing cases were because of no antenatal visit (Figures 1-3).

Pulmonary disease was not significantly found in the 3 groups of patients, while other medical diseases including thyroid diseases, hypertension and diabetes mellitus were significantly found for maternal age $\geq 40$ years (Table 2). Obstetric complications, including severe preeclampsia and gestational diabetes mellitus were differ- 
Table 1. Patients characteristics by maternal age between 2 and 3 groups.

\begin{tabular}{|c|c|c|c|c|c|c|c|}
\hline & \multicolumn{4}{|c|}{ Comparison among 3 groups } & \multicolumn{3}{|c|}{ Comparison between 2 groups } \\
\hline & $\begin{array}{c}\text { Age } \leq 16 \\
(n=1061)\end{array}$ & $\begin{array}{c}\text { Age } 20-29 \\
(n=1100)\end{array}$ & $\begin{array}{c}\text { Age } \geq 40 \\
(n=1100)\end{array}$ & P-value & $\begin{array}{c}\text { Age } \leq 16 \\
(n=1061)\end{array}$ & $\begin{array}{c}\text { Age } \geq 40 \\
(n=1100)\end{array}$ & P-value \\
\hline Maternal age & $\begin{array}{l}15.74 \pm 0.79 \\
(13-16)\end{array}$ & $\begin{array}{l}26 \pm 0.74 \\
(20-29)\end{array}$ & $\begin{array}{c}41.79 \pm 1.69 \\
(40-51)\end{array}$ & $<0.001$ & $\begin{array}{l}15.74 \pm 0.79 \\
(13-17)\end{array}$ & $\begin{array}{c}41.79 \pm 1.69 \\
(40-51)\end{array}$ & $<0.001$ \\
\hline $\begin{array}{l}\text { Body mass index } \\
\qquad\left(\mathrm{kg} / \mathrm{m}^{2}\right) \\
(\text { mean } \pm \mathrm{SD})\end{array}$ & $\begin{array}{c}24.71 \pm 3.88 \\
(16.59-47.61) \\
(\mathbf{n}=\mathbf{9 4 6})\end{array}$ & $\begin{array}{c}27.03 \pm 4.34 \\
(15.80-49.22) \\
(\mathbf{n}=\mathbf{1 0 1 0})\end{array}$ & $\begin{array}{c}27.89 \pm 3.88 \\
(14.51-49.31) \\
(\mathbf{n}=\mathbf{1 0 2 8})\end{array}$ & $<0.001$ & $\begin{array}{c}24.71 \pm 3.88 \\
(16.59-47.61) \\
(\mathbf{n}=\mathbf{9 4 6})\end{array}$ & $\begin{array}{c}27.89 \pm 3.88 \\
(14.51-49.31) \\
(\mathbf{n}=\mathbf{1 0 2 8})\end{array}$ & $<0.001$ \\
\hline Drug abuse & $42(4.0)$ & $24(2.2)$ & $1(0.1)$ & $<0.001$ & $42(4.0)$ & $1(0.1)$ & $<0.001$ \\
\hline No ANC & $45(4.2)$ & $32(2.9)$ & $8(0.7)$ & $<0.001$ & $45(4.2)$ & $8(0.7)$ & $<0.001$ \\
\hline \multicolumn{8}{|l|}{ Gravida } \\
\hline 1 & $1001(94.3)$ & $394(35.8)$ & $171(15.5)$ & & $1001(94.3)$ & $171(15.5)$ & \\
\hline 2 & $52(4.9)$ & $442(40.2)$ & $346(31.5)$ & & $52(4.9)$ & $346(31.5)$ & \\
\hline 3 & $8(0.8)$ & $193(17.5)$ & 309 (28.1) & $<0.001$ & $8(0.8)$ & $309(28.1)$ & $<0.001$ \\
\hline 4 & $0(0)$ & $71(6.5)$ & $274(24.9)$ & & $0(0)$ & $274(24.9)$ & \\
\hline \multicolumn{8}{|l|}{ Parity } \\
\hline 0 & $1038(97.8)$ & $506(46.0)$ & $266(24.2)$ & & $1038(97.8)$ & $266(24.2)$ & \\
\hline 1 & $21(2.0)$ & $431(39.2)$ & $440(40.0)$ & & $21(2.0)$ & $440(40.0)$ & \\
\hline 2 & $2(0.20$ & $132(12.0)$ & 279 (25.4) & $<0.001$ & $2(0.20$ & 279 (25.4) & $<0.001$ \\
\hline 3 & $0(0)$ & $31(2.8)$ & $115(10.5)$ & & $0(0)$ & $115(10.5)$ & \\
\hline \multicolumn{8}{|l|}{ Abortion } \\
\hline 0 & $1020(96.1)$ & $884(80.4)$ & $685(62.3)$ & & $1020(96.1)$ & $685(62.3)$ & \\
\hline 1 & $38(3.6)$ & $170(15.5)$ & $306(27.8)$ & & $38(3.6)$ & $306(27.8)$ & \\
\hline 2 & $3(0.3)$ & $36(3.3)$ & $81(7.4)$ & $<0.001$ & $3(0.3)$ & $81(7.4)$ & $<0.001$ \\
\hline 3 & $0(0)$ & $10(0.9)$ & $28(2.5)$ & & $0(0)$ & $28(2.5)$ & \\
\hline VDRL reactive ${ }^{*}$ & $4(0.4)$ & $4(0.4)$ & $5(0.5)$ & 0.933 & $4(0.4)$ & $5(0.5)$ & 0.775 \\
\hline HBS Ag positive ${ }^{* *}$ & $16(1.5)$ & $33(3.0)$ & $27(2.5)$ & 0.067 & $16(1.5)$ & $27(2.5)$ & 0.112 \\
\hline $\begin{array}{l}\text { Anti-HIV }^{* * *} \\
\text { positive }\end{array}$ & $9(0.8)$ & $5(0.5)$ & $1(0.1)$ & 0.034 & $9(0.8)$ & $1(0.1)$ & 0.010 \\
\hline Hematocrit (\%) & $\begin{array}{l}34.13 \pm 3.39 \\
(24.0-46.0)\end{array}$ & $\begin{array}{l}35.10 \pm 3.36 \\
(19.5-46.0)\end{array}$ & $\begin{array}{l}35.27 \pm 3.03 \\
(26.4-43.0)\end{array}$ & $<0.001$ & $\begin{array}{l}34.13 \pm 3.39 \\
(24.0-46.0)\end{array}$ & $\begin{array}{l}35.27 \pm 3.03 \\
(26.4-43.0)\end{array}$ & 0.048 \\
\hline Anemia & $330(31.1)$ & $215(19.5)$ & $214(19.5)$ & $<0.001$ & $330(31.1)$ & $214(19.5)$ & $<0.001$ \\
\hline Total thalassemia ${ }^{* * * *}$ & $\begin{array}{l}294(29.2) \\
(\mathbf{n}=\mathbf{1 0 0 7})\end{array}$ & $\begin{array}{l}263(24.3) \\
(\mathbf{n}=\mathbf{1 0 8 2})\end{array}$ & $\begin{array}{l}230(21.0) \\
(\mathbf{n}=\mathbf{1 0 9 3})\end{array}$ & $<0.001$ & $\begin{array}{l}294(29.2) \\
(\mathbf{n}=\mathbf{1 0 0 7})\end{array}$ & $\begin{array}{l}230(21.0) \\
(\mathbf{n}=\mathbf{1 0 9 3})\end{array}$ & $<0.001$ \\
\hline $\mathrm{Hb} \mathrm{H}$ disease & $1(0.1)$ & $4(0.4)$ & $4(0.4)$ & 0.415 & $1(0.1)$ & $4(0.4)$ & 0.210 \\
\hline Beta thal trait & $4(0.4)$ & $12(1.1)$ & $12(1.1)$ & 0.140 & $4(0.4)$ & $12(1.1)$ & 0.065 \\
\hline $\mathrm{Hb} \mathrm{E}$ trait & $172(17.1)$ & $149(13.8)$ & $158(14.5)$ & 0.085 & $172(17.1)$ & $158(14.5)$ & 0.09 \\
\hline Alpha thal trait & $123(12.2)$ & $69(6.4)$ & $50(4.6)$ & $<0.001$ & $123(12.2)$ & $50(4.6)$ & $<0.001$ \\
\hline Other types & $8(0.8)$ & $38(3.5)$ & $14(1.3)$ & $<0.001$ & $8(0.8)$ & $14(1.3)$ & 0.346 \\
\hline
\end{tabular}

Data are $\mathrm{n}(\%)$ or mean \pm standard deviation (lower to upper range) unless otherwise specified. P-value represents chi-square statistic comparison among groups for categorical and analysis of variance for continuous variable. ${ }^{*}$ VDRL reactive means positive screening test for syphilis; ${ }^{* *}$ HBS Ag positive means positive screening test for hepatitis B carrier; ${ }^{* * *}$ Anti-HIV positive means positive screening test for the HIV virus antibodies; ${ }^{* * * *}$ Some patients may have both types of thalassemia.

ent with statistical significance between the 2 and 3 groups (Table 2).

Obstetric complications, including placenta previa and preterm labour, were different with statistical significance between the 2 groups (Table 3). However placenta accreta was not detected in the young pregnant age group because most women in this group were in the first pregnancy (Table 3). Preterm and very preterm labour were significantly found in extremely maternal age groups (Table 3).
Medical administrations, including dexamethasone and magnesium sulfate, were significantly detected in the three aged groups of pregnant women but were not different between the extremely young and advanced maternal age groups (Table 4). Gestational age at deliveries, mode of deliveries, fetal numbers, neonatal weights and fetal structural abnormalities were different with statistical significance among the second and third group of patients (Table 4). NICU admission of newborns was different between pregnant women in the 3 groups but 


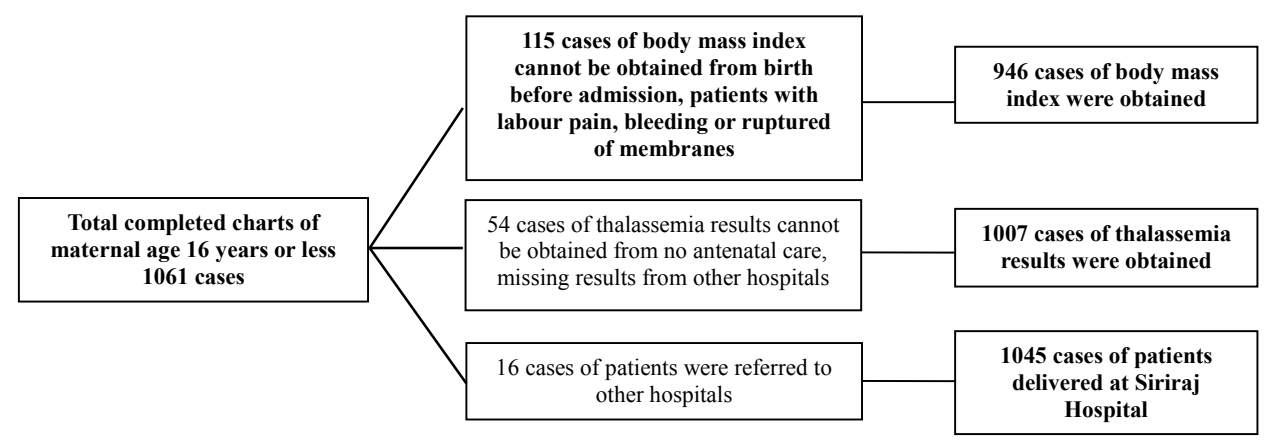

Figure 1. The excluded charts of maternal age 16 years or less were described.

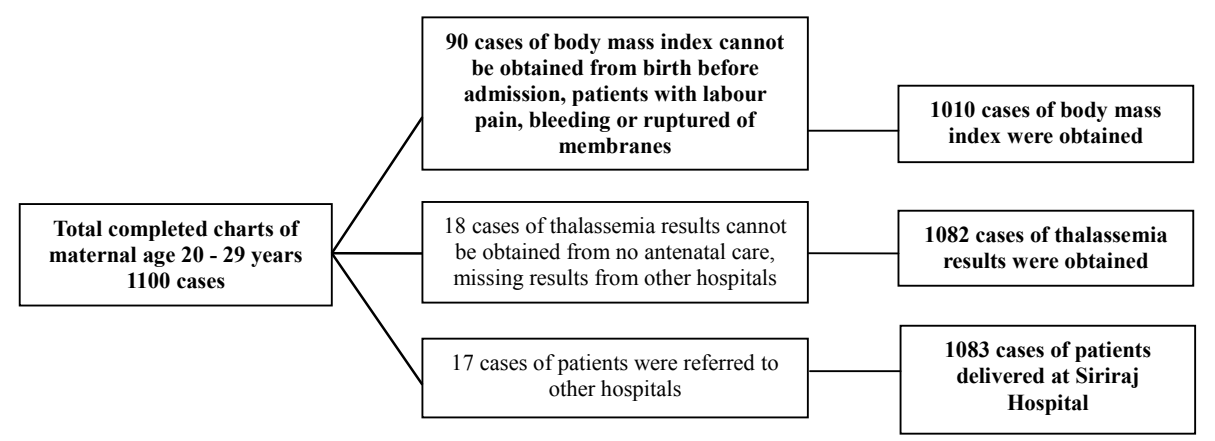

Figure 2. The excluded charts of maternal age 20 - 29 years were described.

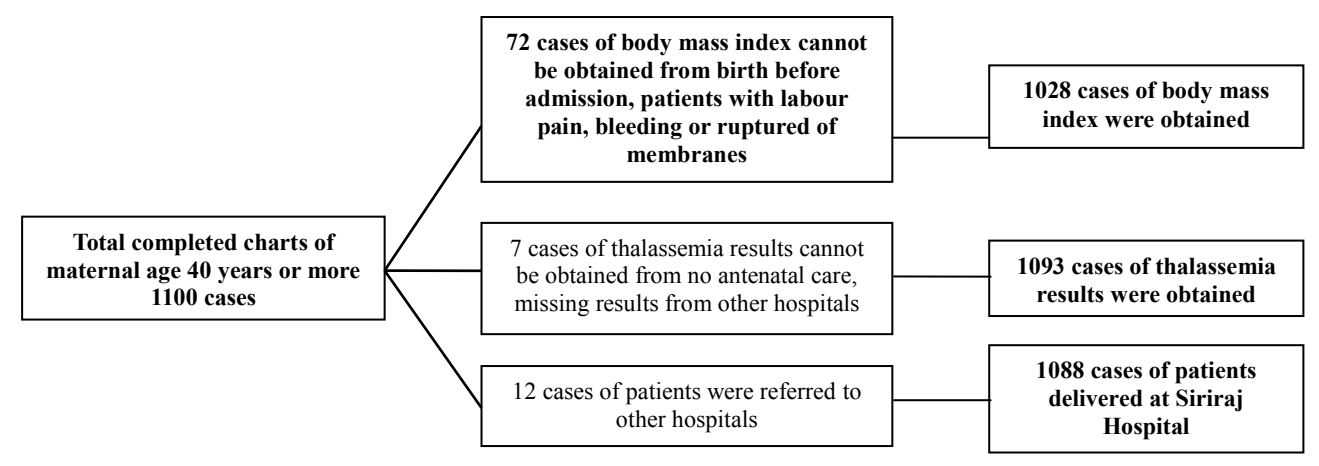

Figure 3. The excluded charts of maternal age $\geq 40$ years were described.

not between extremely maternal age groups (Table 4). Only APGAR score at 1 minute was different with statistical significant between 3 maternal age groups (Table 4).

\section{DISCUSSION}

According to the difference of education and economic status among people in the developing countries, pregnancies for the extremely young and for older women are rising and correlating with adverse pregnancy outcome of both mothers and neonates. Poverty and poor education are usually detected in young maternal age while wealth and high education are usually found in advanced maternal age and also in developed countries [10,11].
Adolescent pregnancy is defined as gestation of women before having reached the full somatic development [12].

The percentage of childbearing adolescent women is varies greatly depending on the cultural, religious, political, economic and other factors of each region [13].

Pregnant women at aged $\leq 16$ years and $\geq 40$ year were recruited in this study because the complications during pregnancy are more common among the pregnant women within these age groups [14-16].

Pregnant women at aged 20 - 29 years were chosen as the control group because pregnant women over 29 years had significant risks of diabetes mellitus, placenta previa, cesarean section, and neonatal intensive care unit (NICU) admission of the newborn [17]. 
Table 2. Underlying medical diseases and medical complications by maternal age between 2 and 3 groups.

\begin{tabular}{|c|c|c|c|c|c|c|c|}
\hline & \multicolumn{4}{|c|}{ Comparison among 3 groups } & \multicolumn{3}{|c|}{ Comparison between 2 groups } \\
\hline & $\begin{array}{c}\text { Age } \leq 16 \\
(n=1061)\end{array}$ & $\begin{array}{c}\text { Age } 20-29 \\
(n=1100)\end{array}$ & $\begin{array}{c}\text { Age } \geq 40 \\
(n=1100)\end{array}$ & P-value & $\begin{array}{l}\text { Age } \leq 16 \\
(n=1061)\end{array}$ & $\begin{array}{c}\text { Age } \geq 40 \\
(n=1100)\end{array}$ & P-value \\
\hline Heart diseases & $7(0.7)$ & $19(1.7)$ & $10(0.9)$ & 0.045 & $7(0.7)$ & $10(0.9)$ & 0.512 \\
\hline Thyroid disease & $1(0.1)$ & $19(1.7)$ & $13(0.2)$ & 0.001 & $1(0.1)$ & $13(0.2)$ & 0.002 \\
\hline Hypertension & $2(0.2)$ & $7(0.6)$ & $21(1.9)$ & $<0.001$ & $2(0.2)$ & $21(1.9)$ & $<0.001$ \\
\hline Pulmonary disease & $12(1.1)$ & $14(1.3)$ & $16(1.5)$ & 0.799 & $12(1.1)$ & $16(1.5)$ & 0.506 \\
\hline Diabetes mellitus & $0(0)$ & $4(0.4)$ & $19(1.7)$ & $<0.001$ & $0(0)$ & $19(1.7)$ & $<0.001$ \\
\hline \multicolumn{8}{|l|}{$\begin{array}{l}\text { Hypertensive } \\
\text { disorders in } \\
\text { pregnancy }\end{array}$} \\
\hline Gestational $\mathrm{HT}^{*}$ & $24(2.3)$ & $28(2.5)$ & $61(5.5)$ & & $24(2.3)$ & $61(5.5)$ & \\
\hline Mild pre-eclampsia & $17(1.6)$ & $18(1.6)$ & $15(1.4)$ & & $17(1.6)$ & $15(1.4)$ & \\
\hline Severe pre-eclampsia & $26(2.5)$ & $12(1.1)$ & $27(2.5)$ & & $26(2.5)$ & $27(2.5)$ & $<0.001$ \\
\hline Elcampsia & $2(0.2)$ & $2(0.2)$ & $0(0)$ & & $2(0.2)$ & $0(0)$ & \\
\hline $\begin{array}{l}\text { Chronic } \mathrm{HT}^{*} \text { with } \\
\text { superimposed } \\
\text { pre-eclampsia }\end{array}$ & $0(0)$ & $0(0)$ & $10(0.9)$ & $<0.001$ & $0(0)$ & $10(0.9)$ & \\
\hline $\begin{array}{l}\text { Gestational diabetes } \\
\mathrm{DM}^{* *} \mathrm{~A} 1\end{array}$ & $1(0.1)$ & $21(1.9)$ & $135(12.3)$ & & $1(0.1)$ & $135(12.3)$ & \\
\hline $\mathrm{DM}^{* *} \mathrm{~A} 2$ at least & $0(0)$ & $5(0.5)$ & $15(1.4)$ & $<0.001$ & $0(0)$ & $15(1.4)$ & $<0.001$ \\
\hline
\end{tabular}

Table 3. Obstetrics complications by maternal age between 2 and 3 groups.

\begin{tabular}{|c|c|c|c|c|c|c|c|}
\hline & \multicolumn{4}{|c|}{ Comparison among 3 groups } & \multicolumn{3}{|c|}{ Comparison between 2 groups } \\
\hline & $\begin{array}{c}\text { Age } \leq 16 \\
(n=1061)\end{array}$ & $\begin{array}{c}\text { Age } 20-29 \\
(n=1100)\end{array}$ & $\begin{array}{c}\text { Age } \geq 40 \\
(n=1100)\end{array}$ & P-value & $\begin{array}{c}\text { Age } \leq 16 \\
(n=1061)\end{array}$ & $\begin{array}{c}\text { Age } \geq 40 \\
(n=1100)\end{array}$ & P-value \\
\hline Abruption placenta & $1(0.1)$ & $1(0.1)$ & $4(0.4)$ & 0.233 & $1(0.1)$ & $4(0.4)$ & 0.193 \\
\hline Low lying placentae & $0(0)$ & $2(0.2)$ & $3(0.3)$ & 0.322 & $0(0)$ & $3(0.3)$ & 0.089 \\
\hline Marginalis & $0(0)$ & $4(0.4)$ & $3(0.3)$ & & $0(0)$ & $3(0.3)$ & \\
\hline Totalis & $0(0)$ & $3(0.3)$ & $20(1.8)$ & $<0.001$ & $0(0)$ & $20(1.8)$ & $<0.001$ \\
\hline Placenta accreta & $0(0)$ & $2(0.2)$ & $5(0.5)$ & 0.071 & $0(0)$ & $5(0.5)$ & 0.028 \\
\hline Very preterm (22 - 32 weeks of gestation) & $34(3.3)$ & $23(2.1)$ & $31(2.8)$ & & $34(3.3)$ & $31(2.8)$ & \\
\hline Preterm (33 - 36 weeks of gestation) & $171(16.4)$ & $96(8.9)$ & $152(14.0)$ & & $171(16.4)$ & $152(14.0)$ & \\
\hline Term & $840(80.4)$ & $964(89.0)$ & $905(83.2)$ & & $840(80.4)$ & $905(83.2)$ & \\
\hline
\end{tabular}

Data are n (\%) unless otherwise specified. P-value represents chi-square statistic comparison among groups for categorical.

About $4.2 \%$ of extremely young maternal age $(\leq 16$ years) had no prenatal care which might be the reason for unintended pregnancy, poverty, ignorance of pregnancy signs and symptoms and lack of knowledge of antenatal care $[14,18]$.

The policy of the national health insurance, the Universal Coverage Project, at a cost of 30 Baht (US\$ 0.75) for the Thai people enables them to get medical care conveniently and thoroughly [19], but the young pregnant women still have many problems involving pregnancy and delivery. Appropriate care will decrease the adverse pregnancy outcomes which result from social rather than physiological conditions [18].

Sexually transmitted diseases including HIV infection and venereal disease were also significantly high in extremely young pregnant women as shown in a previous 
Table 4. Medical administrations in complicated cases and outcomes of deliveries and neonates by maternal age.

\begin{tabular}{|c|c|c|c|c|c|c|c|}
\hline & \multicolumn{3}{|c|}{ Comparison among 3 groups } & \multirow[b]{2}{*}{ P-value } & \multicolumn{3}{|c|}{ Comparison between 2 groups } \\
\hline & $\begin{array}{c}\text { Age } \leq 16 \\
(n=1061)\end{array}$ & $\begin{array}{c}\text { Age } 20-29 \\
(n=1100)\end{array}$ & $\begin{array}{c}\text { Age } \geq 40 \\
(n=1100)\end{array}$ & & $\begin{array}{c}\text { Age } \leq 16 \\
(n=1061)\end{array}$ & $\begin{array}{c}\text { Age } \geq 40 \\
(n=1100)\end{array}$ & P-value \\
\hline Dexamethasone & $23(2.2)$ & $6(0.5)$ & $17(1.5)$ & 0.005 & $23(2.2)$ & $17(1.5)$ & 0.283 \\
\hline Bricanyl & $12(1.1)$ & $3(0.3)$ & $11(1.0)$ & 0.053 & $12(1.1)$ & $11(1.0)$ & 0.767 \\
\hline Magnesium sulfate & $46(4.3)$ & $25(2.3)$ & $58(5.3)$ & 0.001 & $46(4.3)$ & $58(5.3)$ & 0.309 \\
\hline Nifedipine & $0(0)$ & $3(0.3)$ & $3(0.3)$ & 0.235 & $0(0)$ & $3(0.3)$ & 0.089 \\
\hline $\begin{array}{l}\text { Gestational age at deliveries } \\
\text { (ranges, weeks) }\end{array}$ & $\begin{array}{c}37.92 \pm 2.47 \\
(24-43) \\
(\mathrm{n}=1045)\end{array}$ & $\begin{array}{c}38.28 \pm 1.94 \\
(25-43) \\
(\mathrm{n}=1083)\end{array}$ & $\begin{array}{c}37.62 \pm 2.10 \\
(24-42) \\
(\mathrm{n}=1088)\end{array}$ & $<0.001$ & $\begin{array}{c}37.92 \pm 2.47 \\
(24-43) \\
(\mathrm{n}=1045)\end{array}$ & $\begin{array}{c}37.62 \pm 2.10 \\
(24-42) \\
(\mathrm{n}=1088)\end{array}$ & $<0.001$ \\
\hline $\begin{array}{l}\text { Mode of deliveries } \\
\text { Normal labour }\end{array}$ & $865(81.5)$ & $669(60.8)$ & $430(39.1)$ & & $865(81.5)$ & $430(39.1)$ & \\
\hline $\begin{array}{c}\mathrm{F} / \mathrm{E} / \mathrm{V} / \mathrm{E}^{*} \\
\text { Cesarean section }\end{array}$ & $\begin{array}{c}19(1.8) \\
177(16.7)\end{array}$ & $\begin{array}{c}21(1.9) \\
410(37.3)\end{array}$ & $\begin{array}{c}38(3.5) \\
632(57.5)\end{array}$ & $<0.001$ & $\begin{array}{c}19(1.8) \\
177(16.7)\end{array}$ & $\begin{array}{c}38(3.5) \\
632(57.5)\end{array}$ & $<0.001$ \\
\hline $\begin{array}{c}\text { Fetal numbers } \\
1 \\
2 \\
\geq 3\end{array}$ & $\begin{array}{c}1060(99.9) \\
1(0.1) \\
0(0)\end{array}$ & $\begin{array}{c}1088(98.9) \\
12(1.1) \\
0(0)\end{array}$ & $\begin{array}{c}1076(97.8) \\
20(1.8) \\
4(0.4)\end{array}$ & $<0.001$ & $\begin{array}{c}1060(99.9) \\
1(0.1) \\
0(0)\end{array}$ & $\begin{array}{c}1076(97.8) \\
20(1.8) \\
4(0.4)\end{array}$ & $<0.001$ \\
\hline $\begin{array}{l}\text { Neonatal weights } \\
\geq 4000 \mathrm{~g} \\
2500-3999 \mathrm{~g} \\
1500-2499 \mathrm{~g} \\
1000-1499 \mathrm{~g} \\
<1000 \mathrm{~g}\end{array}$ & $\begin{array}{c}5(0.5) \\
871(82.1) \\
161(15.2) \\
21(2.0) \\
3(0.3)\end{array}$ & $\begin{array}{c}18(1.6) \\
967(87.9) \\
108(9.8) \\
3(0.3) \\
4(0.4)\end{array}$ & $\begin{array}{c}28(2.5) \\
911(82.8) \\
146(13.3) \\
10(0.9) \\
5(0.5)\end{array}$ & $<0.001$ & $\begin{array}{c}5(0.5) \\
871(82.1) \\
161(15.2) \\
21(2.0) \\
3(0.3)\end{array}$ & $\begin{array}{c}28(2.5) \\
911(82.8) \\
146(13.3) \\
10(0.9) \\
5(0.5)\end{array}$ & $<0.001$ \\
\hline $\begin{array}{l}\text { Mean neonatal weights } \\
\text { (grams) }\end{array}$ & $\begin{array}{l}2830.77 \pm 481.31 \\
(700-4270)\end{array}$ & $\begin{array}{l}3038.53 \pm 482.23 \\
(720-5280)\end{array}$ & $\begin{array}{l}3012.30 \pm 562.38 \\
\quad(800-4770)\end{array}$ & $<0.001$ & $\begin{array}{l}2830.77 \pm 481.31 \\
(700-4270)\end{array}$ & $\begin{array}{c}3012.30 \pm 562.38 \\
(800-4770)\end{array}$ & $<0.001$ \\
\hline $\mathrm{NICU}^{* *}$ admission & $19(1.8)$ & $9(0.8)$ & $16(1.5)$ & 0.137 & $19(1.8)$ & $16(1.5)$ & 0.536 \\
\hline $\begin{array}{c}\text { Structural abnormalities } \\
\text { CNS } \\
\text { Heart } \\
\text { Abdomen } \\
\text { KUB } \\
\text { Other }\end{array}$ & $\begin{array}{l}6(0.6) \\
5(0.5) \\
1(0.1) \\
0(0) \\
5(0.5)\end{array}$ & $\begin{array}{l}2(0.2) \\
2(0.2) \\
1(0.1) \\
1(0.1) \\
1(0.1)\end{array}$ & $\begin{array}{c}0(0) \\
1(0.1) \\
0(0) \\
0(0) \\
6(0.5)\end{array}$ & 0.029 & $\begin{array}{l}6(0.6) \\
5(0.5) \\
1(0.1) \\
0(0) \\
5(0.5)\end{array}$ & $\begin{array}{c}0(0) \\
1(0.1) \\
0(0) \\
0(0) \\
6(0.5)\end{array}$ & 0.032 \\
\hline $\begin{array}{l}\text { APGAR scores } \\
1 \text { minute } \\
5 \text { minute }\end{array}$ & $\begin{array}{l}8.61 \pm 1.51 \\
(0-10) \\
9.73 \pm 1.11 \\
(0-10)\end{array}$ & $\begin{array}{l}8.68 \pm 1.50 \\
(0-10) \\
9.75 \pm 1.16 \\
(0-10)\end{array}$ & $\begin{array}{c}8.50 \pm 1.63 \\
(0-10) \\
9.70 \pm 1.21 \\
(0-10)\end{array}$ & $\begin{array}{l}0.022 \\
0.551\end{array}$ & $\begin{array}{l}8.61 \pm 1.51 \\
(0-10) \\
9.73 \pm 1.11 \\
(0-10)\end{array}$ & $\begin{array}{c}8.50 \pm 1.63 \\
(0-10) \\
9.70 \pm 1.21 \\
(0-10)\end{array}$ & $\begin{array}{l}0.257 \\
0.262\end{array}$ \\
\hline Stillbirth & $8(0.8)$ & $10(0.9)$ & $9(0.8)$ & 0.923 & $8(0.8)$ & $9(0.8)$ & 0.866 \\
\hline
\end{tabular}

Data are $\mathrm{n}(\%)$ or mean \pm standard deviation (lower to upper range) unless otherwise specified. P-value represents chi-square statistic comparison among groups for categorical and analysis of variance for continuous variable. NICU (neonatal intensive care unit) admission consists of need for ongoing respiratory support, need for oxygen supplementation, continuous positive airway pressure, mechanical ventilation or surfactant administration; ${ }^{*} \mathrm{~F} / \mathrm{E}$, V/E means forceps extraction, vacuum extraction; ${ }^{* *}$ NICU means neonatal intensive care unit; ${ }^{* * *}$ CNS means central nervous system; ${ }^{* * * *}$ KUB means kidney ureter bladder.

study [15]. It is noteworthy that hepatitis B carriers and venereal diseases were not different between extremely young and advanced pregnant women. The possibility of subsequent infection could be from horizontal transmission including sexual intercourse, sharing toothbrushes with partners or directly touching around the wound [20].

Young mothers had a higher rate of anemia with the antenatal hemoglobin concentration $<10 \mathrm{~g} / \mathrm{dL}$ which corresponded to previous study $[14,18]$. This might be the result of poor nutrition during pregnancy [18]. Thalassemia is a genetic disease which is common in Thailand. Alpha thalassemia was found to be higher in pregnant women $\leq 16$ years than in the other groups. This can be explained in that those pregnant women could be from the prevalent community of persons with 
the disease but it can not be explained by clinical evidence.

Medical complications in advanced maternal age including diabetes mellitus, hypertensive disorder in pregnancy and thyroid disease were strongly associated with stillbirth and low birth weight infants and corresponded with the previous studies [21,22]. This can be explained that abnormal thyroid functions and lung diseases were more common among those in the advanced maternal age group because of the maternal exposure to toxic substances or delayed hypersensitivity. The women with clinically congenital heart disease were always needed to be treated before getting pregnant at an advanced age [23]. From subgroup analysis, severe pre-eclampsia was a high obstetric complication in pregnancy of the extremely young and at advanced maternal age, which corresponded to previous studies $[14,15]$.

According to the risks of hypertensive disorder in pregnancy and preterm delivery, it was not surprising that use of steroids to promote lung maturity and magnesium sulfate to prevent convulsion in severe preeclampsia was also high in both extremely aged groups.

Both the extremely young and the advanced maternal age groups had high rates of preterm births and of low birth weight infants which could be from spontaneous preterm and indicated preterm, respectively [21,24]. From subgroup analysis, the complications of abnormal placental attachment including placenta previa, placenta accreta and also placental abruption were significantly different between the 2 groups which were supported by a previous study [25].

Many previous studies also supported observations that the advantage of socio-economic status, high education and marital status were related to lower risks of adverse neonatal outcomes [26,27] Moreover, from subgroup analysis, this study presented evidence that gestational age at deliveries, mode of deliveries, low birth weight and mean neonatal body weight were different between the 2 groups. The cesarean section rate was found to be lower in pregnant women $\leq 16$ years which could be from the low birth weight of infants commonly found in this age group; therefore normal vaginal delivery was possible. However this study did not support a previous study of Thai pregnant adolescents [28].

However, NICU admission and still births were not different between those groups, which could result from indicated preterm relating to hypertensive disorder in those with advanced maternal age of pregnancy.

The large sample size confers sufficient power to evaluate the relation between 3 groups of pregnant women and also rare adverse pregnancy outcomes including neonatal deaths, neonatal abnormalities and NICU admission of infants. The homogeneous population of studied women also supported the findings of this study.
The results of this study finally conclude that pregnancy among the extremely young and those of advanced maternal age entails greater at risk of adverse pregnancy outcomes for both mother and neonates. As a result of less than optimal mental and physical conditions, lack of care and attention to their health, those will be adverse effects on the mother and fetus during pregnancy in young pregnant women [28]. Additionally, recent studies have concluded that advanced maternal age is a predictor of adverse neonatal outcomes relating to underlying diseases such as hypertension, diabetes mellitus and obesity $[29,30]$.

Therefore, education about the effects of maternal age on pregnancy is definitely necessary. Young women must know about how to prevent pregnancy and to have a safe sex in order to reduce adolescent pregnancy. Contraceptive methods should be inserted in the course. However, cultural, social and moral habits among Thai people are still the major issues for dispute. Women with advanced age should realize the risks of underlying diseases and then related affects on pregnancy. The antenatal care plan to reduce complications for both mother and fetus during pregnancy, delivery and the postpartum period must be implemented.

\section{REFERENCES}

[1] Alan Guttmacher Institute (1997) Issues in brief: Risks and realities of early childbearing worldwide. Alan Guttmacher Institute, New York.

[2] World Health Organization (1998) The world health report 1998. Life in the 21st century: A vision for all. WHO, Geneva.

[3] Shrim, A., Ates, S., Mallozzi, A., Brown, R., Ponete, V., Levin, I., et al. (2011) Is young maternal age really a risk factor for adverse pregnancy outcome in a Canadian tertiary referral hospital? Journal of Pediatric and Adolescent Gynecology, 24, 218-222. doi:10.1016/j.jpag.2011.02.008

[4] Cunningham, F.G., Levono, K.L., Bloom, S.L., Hauth, J.C., ROouse, D.J. and Spong, C.Y. (2010) Chapter 5: Maternal physiology. In: Cunningham, F.G., Levono, K.L., Bloom, S.L., Hauth, J.C., Rouse, D.J. and Spong, C.Y., Eds., Williams Obstetrics, 23rd Edition, McGrawHill Companies, New York, 115.

[5] Wattaganara, T. (2010) Clinical practice guideline for management of hypertensive disorder in pregnancy 2010. Department of obstetrics and gynaecology. Mahidol University, Bangkok.

[6] Sansaneewittayakul, P. (2010) Clinical practice guideline for management of diabetes mellitus in pregnancy 2010 . Department of obstetrics and gynaecology. Mahidol University, Bangkok.

[7] Cunningham, F.G., Levono, K.L., Bloom, S.L., Hauth, J.C., ROouse, D.J. and Spong, C.Y. (2010) Chapter 35: Obstetricial hemorrhage. In: Cunningham, F.G., Levono, 
K.L., Bloom, S.L., Hauth, J.C., Rouse, D.J. and Spong, C.Y., Eds., Williams Obstetrics, 23rd Edition, McGrawHill Companies, New York, 809.

[8] Chawanpaiboon, S. (2010) Clinical practice guideline for management of preterm labour 2010. Mahidol University, Bangkok.

[9] Cunningham, F.G., Levono, K.L., Bloom, S.L., Hauth, J.C., Rouse, D.J. and Spong, C.Y. (2010) Chapter 1: Overview of obstetrics. In: Cunningham, F.G., Levono, K.L., Bloom, S.L., Hauth, J.C., Rouse, D.J. and Spong, C.Y., Eds., Williams Obstetrics, 23rd Edition, McGrawHill Companies, New York, 3.

[10] Domenico, D.M. and Jones, K.H. (2007) Adolescent pregnancy in America: Causes and responses. Journal for Vocational Special Needs Education, 30, 4-12.

[11] Simchen, M.J., Yinon, Y., Moran, O., Schiff, E. and Sivan, E. (2006) Pregnancy outcome after age 50. Obstetrics \& Gynecology, 108, 1084-1088. doi:10.1097/01.AOG.0000240139.46018.bd

[12] Scholl, T.O., Hediger, M.L., Salmon, R.W., Belsky, D.H. and Ances, I.G. (1989) Association between gynecological age and preterm birth. Paediatric and Perinatal Epidemiology, 3, 357-366. doi:10.1111/j.1365-3016.1989.tb00524.x

[13] Paranjothy, S., Broughton, H., Adappa, R. and Fone, D. (2008) Teenage pregnancy: Who suffers? Archives of Disease in Childhood, 94, 239-245. doi:10.1136/adc.2007.115915

[14] Phipps, M.G. and Sowers, M. (2002) Defining early adolescent childbearing. American Journal of Public Health, 92, 125-128.

[15] Kurth, F., Bélard, S., Mombo-Ngoma, G., Schuster, K., Adegnika, A.A. and Bouyou-Akotet, M.K. (2010) Adolescence as risk factor for adverse pregnancy outcome in Central Africa: A cross-sectional study. PLOS ONE, 5, e14367. doi:10.1371/journal.pone.0014367

[16] Carolan, M. and Frankowska, D. (2011) Advanced maternal age and adverse perinatal outcome: A review of the evidence. Midwifery, 27, 793-801. doi:10.1016/j.midw.2010.07.006

[17] Koo, J.Y., Ryu, M.H., Yang, H.J., Lim, H.J., Lee, E.J., Kim, Y.M., et al. (2012) Pregnancy outcomes according to increasing maternal age. Taiwanese Journal of Obstetrics and Gynecology, 51, 60-65. doi:10.1016/j.tjog.2012.01.012

[18] Jolly, M.C., Sebire, N., Harris, J., Robinson, S. and Regan, L. (2000) Obstetric risks of pregnancy in women less than 18 years old. Obstetrics and Gynecology, 96, 962-966. doi:10.1016/S0029-7844(00)01075-9

[19] National Health Security Office of Thailand (2001) The Universal Coverage Project. http://www.nhso.go.th
[20] Martinson, F.E.A., Weigle, K.A., Royce, R.A., Weber, D.J., Suchindran, C.M. and Lemon, S.M. (1998) Risk factors for horizontal transmission of hepatitis B virus in a rural district in Ghana. American Journal of Epidemiology, 147, 478-487. doi:10.1093/oxfordjournals.aje.a009474

[21] Montan, S. (2007) Increased risk in the elderly parturient. Current Opinion in Obstetrics and Gynecology, 19, 110112. doi:10.1097/GCO.0b013e3280825603

[22] Fretts, R.C. (2005) Etiology and prevention of stillbirth. American Journal of Obstetrics and Gynecology, 193, 1923-1935. doi:10.1016/j.ajog.2005.03.074

[23] Helgadottr, L.B., Skjeldestad, F.E., Jacobsen, A.F., Sandset, P.M. and Jacobsen, E.M. (2011) Incidence and risk factors of fetal death in Norway: A case-control study. Acta Obstetricia et Gynecologica Scandinavica, 90, 390397. doi:10.1111/j.1600-0412.2011.01079.x

[24] Ziadeh S. (2001) Obstetric outcome of teenage pregnancies in North Jordan. Archives of Gynecology and Obstetrics, 265, 26-29. doi:10.1007/s004040000121

[25] Cleary-Goldman, J., Malone, F.D., Vidaver, J., Ball, R.H., Nyberg, D.A., Comstock, C.H., et al. (2005) Impact of maternal age on obstetric outcome. Obstetrics \& Gynecology, 105, 983-990. doi:10.1097/01.AOG.0000158118.75532.51

[26] Moser, K. (2008) Report: Infant and perinatal mortality by social and biological factors, 2007. Health Statistics Quarterly, 40, 61-65. www.statistics.gov.uk/statbase/Product.asp?vlnk=6725S

[27] Goy, J., Dodds, L., Rosenberg, M.W. and King, W.D. (2008) Health-risk behaviours: Examining social disparities in the occurrence of stillbirth. Paediatric and Perinatal Epidemiology, 22, 314-320. doi:10.1111/j.1365-3016.2008.00947.x

[28] Thato, S., Rachukul, S. and Sopajaree, C. (2007) Obstetrics and perinatal outcomes of Thai pregnant adolescents: A retrospective study. International Journal of Nursing Studies, 44, 1158-1164. doi:10.1016/j.ijnurstu.2006.05.016

[29] Yuan, W., Steffensen, F.H., Nielsen, G.L., Møller, M., Olsen, J. and Sørensen, H.T. (2000) A population-based cohort study of birth and neonatal outcome in older primipara. International Journal of Gynecology \& $\mathrm{Ob}$ stetrics, 68, 113-118. doi:10.1016/S0020-7292(99)00181-2

[30] Panagopoulos, P., Economou, A., Tagia, M., Siropoulos, N., Doulia-Anagnostaki, P. and Katsetos, C. (2006) Pregnancy outcome in nulliparous women at age $>35$ in comparison to younger nulliparous women. Giornale Italiano di Ostetricia e Ginecologia, 28, 479-482. 